\title{
IS THE POST-AGB STAR SAO 40039 MILDLY HYDROGEN-DEFICIENT?
}

\author{
S. Sumangala RaO ${ }^{1}$, Gajendra Pandey $^{1}$, David L. Lambert ${ }^{2}$, And Sunetra Giridhar ${ }^{1}$ \\ ${ }^{1}$ Indian Institute of Astrophysics, Bengaluru-560034, India; \\ sumangala@iiap.res.in, pandey@iiap.res.in, giridhar@iiap.res.in \\ ${ }^{2}$ W. J. McDonald Observatory, The University of Texas, Austin, TX 78712, USA; d1l@ astro.as.utexas.edu \\ Received 2011 June 22; accepted 2011 June 30; published 2011 July 19
}

\begin{abstract}
We have conducted an LTE abundance analysis for SAO 40039, a warm post-AGB star whose spectrum is known to show surprisingly strong He I lines for its effective temperature and has been suspected of being $\mathrm{H}$-deficient and He-rich. High-resolution optical spectra are analyzed using a family of model atmospheres with different $\mathrm{He} / \mathrm{H}$ ratios. Atmospheric parameters are estimated from the ionization equilibrium set by neutral and singly ionized species of $\mathrm{Fe}$ and $\mathrm{Mg}$, the excitation of $\mathrm{Fe} \mathrm{I}$ and $\mathrm{Fe}$ II lines, and the wings of the Paschen lines. On the assumption that the $\mathrm{He}$ I lines are of photospheric and not chromospheric origin, a $\mathrm{He} / \mathrm{H}$ ratio of approximately unity is found by imposing the condition that the adopted $\mathrm{He} / \mathrm{H}$ ratio of the model atmosphere must equal the ratio derived from the observed He I triplet lines at 5876, 4471, and $4713 \AA$, and singlet lines at 4922 and $5015 \AA$. Using the model with the best-fitting atmospheric parameters for this $\mathrm{He} / \mathrm{H}$ ratio, SAO 40039 is confirmed to exhibit mild dust-gas depletion, i.e., the star has an atmosphere deficient in elements of high condensation temperature. The star appears to be moderately metal-deficient with $[\mathrm{Fe} / \mathrm{H}]=-0.4$ dex. But the star's intrinsic metallicity as estimated from $\mathrm{Na}$, $\mathrm{S}$, and $\mathrm{Zn}$, elements of a low condensation temperature, is $[\mathrm{Fe} / \mathrm{H}]_{\mathrm{o}} \simeq-0.2\left([\mathrm{Fe} / \mathrm{H}]_{\mathrm{o}}\right.$ refers to the star's intrinsic metallicity). The star is enriched in $\mathrm{N}$ and perhaps $\mathrm{O}$ as well, changes reflecting the star's AGB past and the event that led to He enrichment.
\end{abstract}

Key words: stars: abundances - stars: atmospheres - stars: evolution - stars: fundamental parameters

Online-only material: machine-readable table

\section{INTRODUCTION}

SAO 40039, also known as IRAS $05040+4820$ and BD $+48^{\circ} 1220$, is judged by several criteria to be a post-AGB star: its spectral type (A4Ia), its position in the IRAS color-color diagram, and the double-peaked spectral energy distribution indicating a detached youthful cold dust shell around the central star. Using VBRIJHK photometry and adopting a simple dust shell model, Fujii et al. (2002) find a dynamical age of 2460 years for the dust shell with dust at $97 \mathrm{~K}$ and estimate the star to have a core mass of $0.55 M_{\odot}$, indicating a low-mass progenitor for this PAGB star.

Klochkova et al. (2007) have presented an abundance analysis of SAO 40039 based on high-resolution spectra with a wavelength coverage of 4500-6760 A. They have also discussed the star's radial velocity changes and also time-dependent differential velocity shifts between different classes of absorption lines. Their spectra showed a variable $\mathrm{H} \alpha$ line with two emission components. Variable emission was also reported in $\mathrm{H} \beta$ and in some lines of $\mathrm{Si}$ II, $\mathrm{Fe}$ I, and $\mathrm{Fe}$ II. But, in particular, these authors pointed out the abnormal strength of the He I $5876 \AA$ absorption feature in this star with a "low" effective temperature of about $8000 \mathrm{~K}$. Their abundance analyses conducted with model atmospheres computed for a normal $\mathrm{He} / \mathrm{H}$ ratio $(=0.1)$ gave an $\mathrm{He} / \mathrm{H}$ ratio of 0.7 .

Detection of He I lines in absorption in several A- and F-type PAGB stars has been reported previously. The 4471 A line was found by Waelkens et al. (1992) in HD 44179, the central star of the Red Rectangle. HD 187885, a PAGB star with a high C/O ratio and an enrichment of $s$-process elements, shows the He I 5876 Å line (Van Winckel et al. 1996). Klochkova et al. (2002) reported five $\mathrm{He}$ I lines in HD 331319. Detection of He I lines in A- and F-type supergiants suggests either a He enrichment, if the lines are of photospheric origin, or a contribution from a thick hot chromosphere of a presumably normal He abundance. The above studies adopt the assumption of a photospheric origin and report the photosphere to be moderately H-poor and He-rich. However, these analyses use model atmospheres computed for a normal $\mathrm{He} / \mathrm{H}$ ratio and do not iterate on the construction of the model atmosphere and the abundance analysis to obtain agreement between the input and output $\mathrm{He} / \mathrm{H}$ ratio.

Our goal in producing a fresh analysis of SAO 40039 is to iterate to find the self-consistent $\mathrm{He} / \mathrm{H}$ ratio. In undertaking this analysis, we also adopt the assumption that the $\mathrm{He}$ I lines originate in an He-rich photosphere and are not chromospheric in origin.

\section{OBSERVATIONS AND DATA REDUCTION}

Spectra of SAO 40039 were obtained on the nights of 2007 November 5 and 2011 February 21 at the W. J. McDonald Observatory with the $2.7 \mathrm{~m}$ Harlan J. Smith Telescope and the Tull Coudé Spectrograph (Tull et al. 1995). The spectra taken at these epochs were found to be similar. The spectra correspond to a resolving power of $60,000\left(5 \mathrm{~km} \mathrm{~s}^{-1}\right)$. Reduction of the raw data was performed with the IRAF ${ }^{3}$ software package.

An additional spectrum was obtained on 2011 January 28 using the echelle spectrometer at Vainu Bappu Observatory (VBO) in Kavalur, India, giving a resolution of about 28,000 $\left(10 \mathrm{~km} \mathrm{~s}^{-1}\right)$ in slitless mode (Rao et al. 2005). Since the projected rotational velocity of the star is about $15 \mathrm{~km} \mathrm{~s}^{-1}$, the lower resolution of the VBO spectrum does not affect the stellar absorption profiles and hence our measured equivalent widths and calculated abundances.

\footnotetext{
3 The IRAF software is distributed by the National Optical Astronomy Observatories under contract with the National Science Foundation.
} 

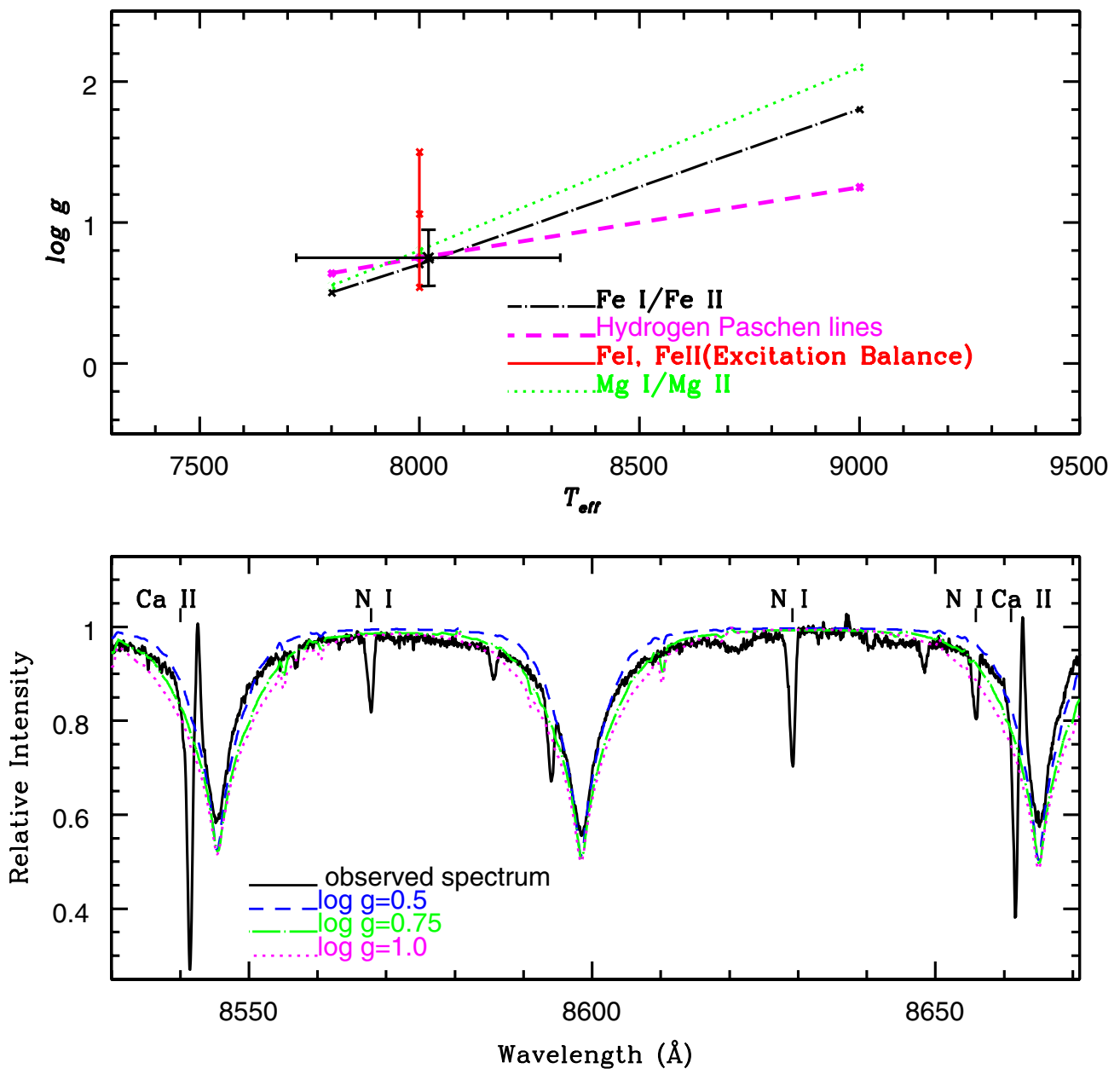

Figure 1. Top panel shows the loci satisfying the ionization balance-see keys on the figure. The locus satisfying the Hi Paschen lines is shown by the dashed line. The solid line represents the excitation balance of both Fe I and Fe II. The cross shows the derived stellar parameters. The bottom panel shows the observed and synthesized profiles of Paschen lines of $\mathrm{HI}$ in the $8600 \AA$ region for $T_{\text {eff }}=8000 \mathrm{~K}, \mathrm{He} / \mathrm{H}=1.0$, and $\log g=0.5,0.75$, and 1.0_-see keys on the figure.

\section{ABUNDANCE ANALYSIS}

Our spectra resemble closely those described by Klochkova et al. (2007) with regard to emission in Balmer lines, the complex structure of the $\mathrm{Na} \mathrm{D}$ lines and variable profiles of the metallic lines, and the equivalent widths of lines unaffected by obvious emission. For our analysis we have used only clean, unblended, and symmetric absorption lines.

We have compared the equivalent widths of lines common between our spectra and those of Klochkova et al. (2007). A small systematic difference possibly caused by differences in resolution is discernible, but it appears that there were no major variations in the atmospheric parameters at these epochs. This is also supported by the fact that the average radial velocity of lines in our spectra $\left(-12 \mathrm{~km} \mathrm{~s}^{-1}\right)$ lies within the range as reported by Klochkova et al. (2007) in their spectra ( -7 to $-15 \mathrm{~km} \mathrm{~s}^{-1}$ ).

Abundance analysis was done first on the assumption that the atmosphere has a normal $\mathrm{He} / \mathrm{H}$ ratio. Model atmospheres for a normal $\mathrm{He} / \mathrm{H}$ ratio $(\mathrm{He} / \mathrm{H}=0.1)$ were taken from the Kurucz database. ${ }^{4}$ Atmospheres of different $\mathrm{He} / \mathrm{H}$ ratios were computed by the code STERNE (Jeffery et al. 2001). The LTE spectrum synthesis code MOOG (2009 version) by Sneden (1973) was used with the grid of model atmospheres taken from the Kurucz

\footnotetext{
4 http://kurucz.harvard.edu/grids.html
}

database as mentioned above. The LTE code SPECTRUM (Jeffery et al. 2001) was used with the STERNE models. ${ }^{5}$

The procedure begins with the determination of the atmospheric parameters from the spectrum, continues with the abundance analysis for $\mathrm{He}$ and other elements and ends when the model of a certain $\mathrm{He} / \mathrm{H}$ ratio reproduces the observed $\mathrm{He} \mathrm{I}$ lines.

\subsection{Method for Determining the Stellar Parameters}

First, we estimated the microturbulent velocity $\left(\xi_{t}\right)$ using Fe II lines with a small range in lower excitation potentials (LEPs, $2.6-3.2 \mathrm{eV}$ ) as these lines have a good range in their equivalent widths thereby reducing any temperature dependence of the estimated $\xi_{t}$. The $\xi_{t}$ is found from the standard requirement that the abundance be independent of the measured equivalent width. The effective temperature $\left(T_{\text {eff }}\right)$ was estimated by the requirement that the abundance of a given species be independent of a line's LEP. This step was conducted independently for Fe I and Fe II lines as they are both well represented in the spectrum and show a range in their LEPs. Finally, the surface gravity

\footnotetext{
5 Note that the stellar parameters and the abundances derived by adopting the Kurucz models and the code MOOG for $\mathrm{He} / \mathrm{H}=0.1$ are in excellent agreement with results from the STERNE models and the LTE code SPECTRUM, as well as for $\mathrm{He} / \mathrm{H}=0.1$.
} 


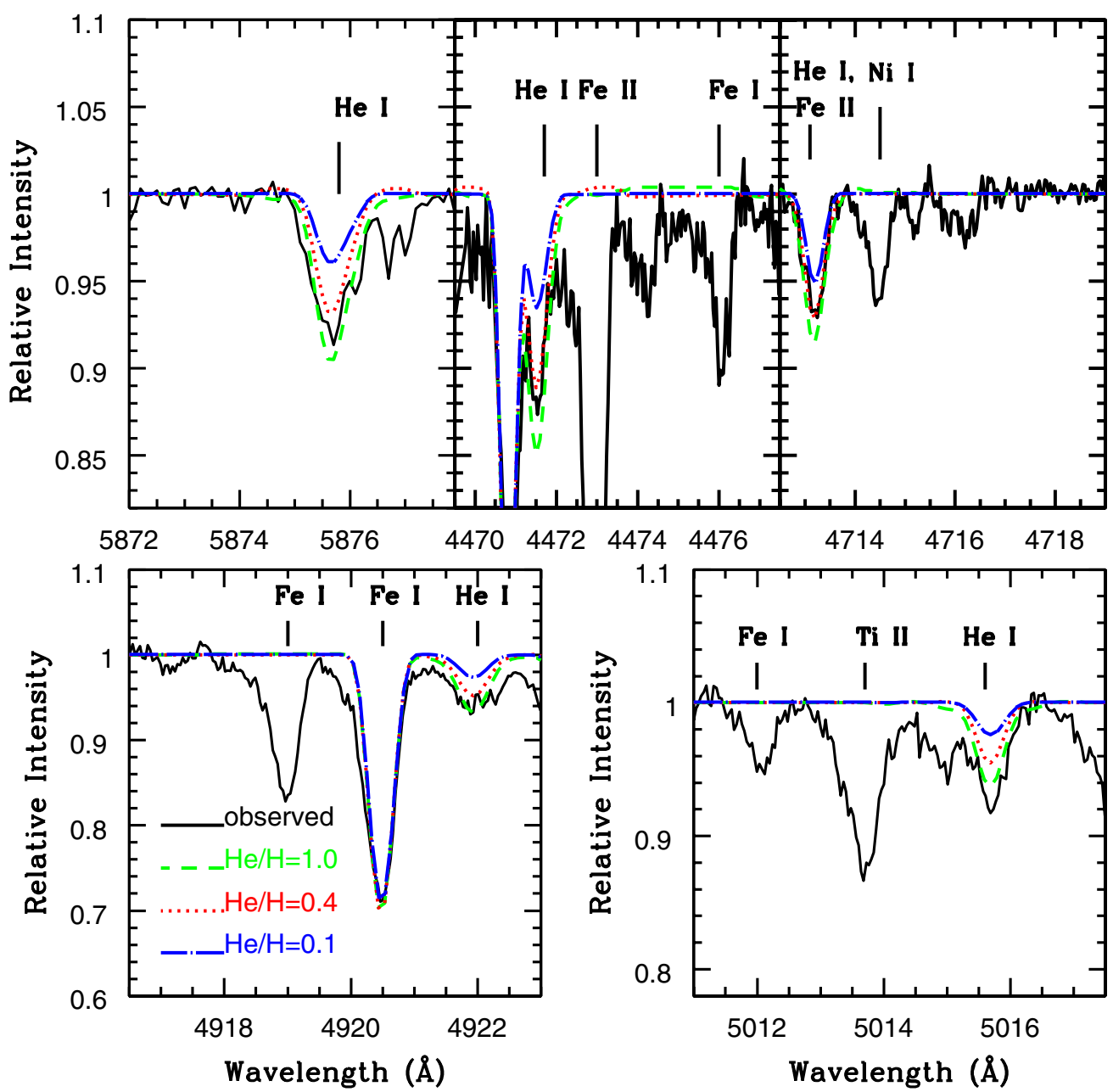

Figure 2. Observed and synthesized He I profiles for the triplet lines at 5876, 4471, and $4713 \AA$ and for the singlet lines at 4922 and $5015 \AA$ for SAO 40039 using models with $T_{\text {eff }}=8000 \mathrm{~K}, \log g=0.75$ for $\mathrm{He} / \mathrm{H}=1.0,0.4$, and 0.1 -see keys on the figure.

$(\log g)$ was estimated by the condition that there be ionization balance between the neutral and the singly ionized Fe lines. This condition defines a locus in the $\left(T_{\text {eff }}, \log g\right)$ plane and the $T_{\text {eff }}$ derived from $\mathrm{Fe}$ I and Fe II breaks the degeneracy. This exercise is repeated for grids of model atmospheres with $\mathrm{He} / \mathrm{H}$ ratios of 10/90, 30/70, and 50/50.

A microturbulence $\xi_{t}=4.8 \pm 1.0 \mathrm{~km} \mathrm{~s}^{-1}$ is found for all models with acceptable effective temperature and surface gravity and the value is insensitive to the $\mathrm{He} / \mathrm{H}$ ratio of the models. The effective temperature $T_{\text {eff }}$ is found to be $8000 \pm 300 \mathrm{~K}$ from $26 \mathrm{Fe} \mathrm{I}$ and $20 \mathrm{Fe}$ II lines. Within the $300 \mathrm{~K}$ uncertainty, the temperature is independent of surface gravity over a considerable range and is not sensitive to the $\mathrm{He} / \mathrm{H}$ ratio of the model atmosphere.

The $T_{\text {eff }}, \log g$ locus found from the ionization balance for $\mathrm{Fe}$ is illustrated in Figure 1 for the model atmosphere grid with $\mathrm{He} / \mathrm{H}=50 / 50$. With $T_{\text {eff }}=8000 \pm 300 \mathrm{~K}$ from the excitation of $\mathrm{Fe} \mathrm{I}$ and $\mathrm{Fe}$ II, the $\log g=0.75 \pm 0.25 \mathrm{cgs}$ is found. The surface gravity changes slightly with $\mathrm{He} / \mathrm{H}$ running from 0.94 for $\mathrm{He} / \mathrm{H}=10 / 90$ through 0.83 for $\mathrm{He} / \mathrm{H}=30 / 70$ to the above result for $\mathrm{He} / \mathrm{H}=50 / 50$. A second locus is provided by $\mathrm{Mg}$ I and $\mathrm{Mg}$ II lines-see the top panel of Figure 1. The $\mathrm{Mg}$ and Fe loci are in good agreement. A third locus is offered by the Paschen lines (see below).

\subsection{The He/H Ratio}

Our spectra confirm the presence of the D3 $5876 \AA$ He I line with an equivalent width very similar to that reported by Klochkova et al. (2007). The strength of this line suggests that several other lines should be present unless the excitation of the D3 line is highly peculiar. Our search also provided the additional triplets (4471 and $4713 \AA$ ) and two singlet lines (4921 and $5015 \AA$ ). Since the He I at $4713 \AA$ is blended with the Fe II line, its contribution has been included, while synthesizing the He I profile at $4713 \AA$ as shown in the Figure 2 we get a consistent $\mathrm{He} / \mathrm{H}$ ratio of approximately unity. Other possible $\mathrm{He} \mathrm{I}$ lines are predicted to be below the detection limit, or blended with other lines or inaccessible due to inter-order gaps.

The He I lines were computed for model atmospheres with $\left(T_{\text {eff }}, \log g, \xi_{t}\right)=(8000,0.75,4.8)$ and with the appropriate model for the $\mathrm{He} / \mathrm{H}$ ratios: 0.1 (normal, solar abundance), 0.4 $(30 / 70)$, and $1.0(50 / 50)$. The synthesized profiles are convolved with the instrumental and the stellar rotation profiles before matching with the observed spectrum as described in Pandey (2006). The data for computing the He I profiles were taken from Jeffery et al. (2001). A projected rotational velocity of about $13-16 \mathrm{~km} \mathrm{~s}^{-1}$ is estimated by using unblended moderately strong lines. As shown in Figure 2, the best fits to the observed 
Table 1

Comparison between Abundances Estimated with MOOG Using the $\mathrm{He} / \mathrm{H}=0.1$ Model and the Abundances Computed with SPECTRUM Using the Hydrogen-deficient $(\mathrm{He} / \mathrm{H}=1.0)$ Model

\begin{tabular}{|c|c|c|c|c|c|c|c|c|c|c|}
\hline \multirow[t]{2}{*}{ Species } & \multirow[t]{2}{*}{$\log \epsilon_{\odot}$} & \multicolumn{3}{|c|}{$\mathrm{MOOG}^{\mathrm{a}}$} & \multicolumn{3}{|c|}{ SPECTRUM $^{\mathrm{b}}$} & \multicolumn{3}{|c|}{ SPECTRUM $^{\mathrm{c}}$} \\
\hline & & $\log \epsilon$ & {$[\mathrm{X} / \mathrm{Fe}]$} & $n$ & $\log \epsilon$ & {$[\mathrm{X} / \mathrm{Fe}]$} & $n$ & $\Delta^{\mathrm{d}}$ & {$[\mathrm{X} / \mathrm{Fe}]$} & $n$ \\
\hline $\mathrm{HI}_{\mathrm{I}}$ & 12.00 & 12.00 & $\ldots$ & $\ldots$ & 11.45 & $\ldots$ & $\ldots$ & $\ldots$ & $\ldots$ & $\ldots$ \\
\hline He I & 10.98 & 10.98 & $\ldots$ & $\ldots$ & 11.45 & $\ldots$ & $\ldots$ & 0.2 & $\ldots$ & $\ldots$ \\
\hline $\mathrm{Li} I$ & 3.26 & 4.03 & $<+0.68 \pm 0.00$ & 1 & 3.50 & $<+0.67 \pm 0.00$ & 1 & $\ldots$ & $\ldots$ & \\
\hline $\mathrm{C}_{\mathrm{I}}$ & 8.43 & 8.78 & $+0.26 \pm 0.16$ & 21 & 8.24 & $+0.24 \pm 0.16$ & 21 & 0.3 & $+0.27 \pm 0.18$ & 9 \\
\hline $\mathrm{N}_{\mathrm{I}}$ & 7.83 & 9.05 & $+1.13 \pm 0.25$ & 9 & 8.48 & $+1.08 \pm 0.20$ & 9 & 0.1 & $+1.32 \pm 0.04$ & 2 \\
\hline $\mathrm{O}_{\mathrm{I}}$ & 8.69 & 9.47 & $+0.69 \pm 0.15$ & 8 & 8.93 & $+0.67 \pm 0.14$ & 8 & 0.1 & $+0.76 \pm 0.14$ & 2 \\
\hline $\mathrm{Na} I$ & 6.24 & 6.58 & $+0.25 \pm 0.10$ & 2 & 6.08 & $+0.27 \pm 0.07$ & 2 & 0.3 & $\ldots$ & $\ldots$ \\
\hline $\mathrm{Mg}_{\mathrm{I}}$ & 7.60 & 7.76 & $+0.07 \pm 0.31$ & 4 & 7.13 & $-0.04 \pm 0.18$ & 4 & 0.4 & $-0.26 \pm 0.11$ & 2 \\
\hline Mg II & 7.60 & 7.78 & $+0.09 \pm 0.15$ & 4 & 7.12 & $-0.05 \pm 0.15$ & 4 & 0.1 & $-0.10 \pm 0.03$ & 3 \\
\hline $\mathrm{Al} \mathrm{I}$ & 6.45 & 5.95 & $-0.59 \pm 0.01$ & 2 & 5.32 & $-0.70 \pm 0.03$ & 2 & 0.4 & $\ldots$ & $\ldots$ \\
\hline Si II & 7.51 & 7.90 & $+0.30 \pm 0.16$ & 3 & 7.37 & $+0.29 \pm 0.13$ & 3 & 0.2 & $+0.31 \pm 0.00$ & 1 \\
\hline S I & 7.12 & 7.53 & $+0.32 \pm 0.01$ & 2 & 7.01 & $+0.32 \pm 0.02$ & 2 & 0.3 & $\ldots$ & $\ldots$ \\
\hline $\mathrm{CaI}$ & 6.34 & 6.18 & $-0.25 \pm 0.12$ & 2 & 5.55 & $-0.36 \pm 0.16$ & 2 & $\ldots$ & $\ldots$ & $\ldots$ \\
\hline Ca II & 6.34 & 6.21 & $-0.22 \pm 0.00$ & 1 & 5.64 & $-0.27 \pm 0.00$ & 1 & 0.4 & $-0.36 \pm 0.00$ & 1 \\
\hline Sc II & 3.15 & 2.89 & $-0.35 \pm 0.17$ & 5 & 2.42 & $-0.30 \pm 0.15$ & 5 & 0.3 & $-0.05 \pm 0.16$ & 2 \\
\hline Ti II & 4.95 & 4.83 & $-0.21 \pm 0.23$ & 20 & 4.32 & $-0.20 \pm 0.20$ & 20 & 0.3 & $-0.24 \pm 0.16$ & 12 \\
\hline V II & 3.93 & 4.21 & $+0.19 \pm 0.04$ & 2 & 3.74 & $+0.24 \pm 0.03$ & 2 & 0.2 & $\ldots$ & $\ldots$ \\
\hline $\mathrm{Cr}$ & 5.64 & 5.62 & $-0.11 \pm 0.13$ & 3 & 5.11 & $-0.10 \pm 0.10$ & 3 & 0.4 & $-0.06 \pm 0.23$ & 2 \\
\hline Cr II & 5.64 & 5.83 & $+0.10 \pm 0.11$ & 9 & 5.33 & $+0.12 \pm 0.10$ & 9 & 0.2 & $+0.09 \pm 0.06$ & 4 \\
\hline Mn II & 5.43 & 5.73 & $+0.21 \pm 0.00$ & 1 & 5.24 & $+0.24 \pm 0.00$ & 1 & $\ldots$ & $\ldots$ & \\
\hline $\mathrm{Fe}_{\mathrm{I}}$ & 7.50 & 7.54 & $-0.05 \pm 0.18$ & 26 & 7.02 & $-0.05 \pm 0.17$ & 26 & 0.4 & $-0.01 \pm 0.19$ & 7 \\
\hline Fe II & 7.50 & 7.65 & $+0.06 \pm 0.19$ & 20 & 7.13 & $+0.06 \pm 0.17$ & 20 & 0.2 & $+0.01 \pm 0.14$ & 9 \\
\hline Ni I & 6.22 & 6.16 & $-0.15 \pm 0.15$ & 4 & 5.63 & $-0.16 \pm 0.14$ & 4 & 0.3 & $\ldots$ & \\
\hline $\mathrm{Zn}$ I & 4.56 & 4.81 & $+0.16 \pm 0.30$ & 2 & 4.28 & $+0.15 \pm 0.23$ & 2 & 0.4 & $\ldots$ & \\
\hline Sr II & 2.87 & 3.01 & $+0.05 \pm 0.07$ & 2 & 2.38 & $-0.06 \pm 0.03$ & 2 & $\ldots$ & $\ldots$ & \\
\hline $\mathrm{Zr}$ II & 2.58 & 2.13 & $-0.54 \pm 0.08$ & 2 & 1.65 & $-0.50 \pm 0.04$ & 2 & 0.3 & $\ldots$ & \\
\hline Ba II & 2.18 & 1.85 & $-0.42 \pm 0.15$ & 2 & 1.30 & $-0.45 \pm 0.11$ & 2 & $\ldots$ & $-0.35 \pm 0.00$ & 1 \\
\hline Eu II & 0.52 & 0.40 & $<-0.10 \pm 0.00$ & 1 & 0.15 & $<+0.06 \pm 0.00$ & 1 & $\ldots$ & $\ldots$ & \\
\hline
\end{tabular}

Notes.

a MOOG abundances computed using the $\mathrm{He} / \mathrm{H}=0.1$ model for the McDonald spectra.

b SPECTRUM abundances computed using the $\mathrm{He} / \mathrm{H}=1.0$ model for the McDonald spectra.

${ }^{c}$ SPECTRUM abundances computed using the $\mathrm{He} / \mathrm{H}=1.0$ model for the VBO spectra.

${ }^{\mathrm{d}} \triangle$ corresponds to the square root of the sum of the squares of the abundance errors due to uncertainties in the stellar parameters: $\triangle T_{\text {eff }}, \triangle$ log $g$, and $\triangle \xi_{t}$.

He I profiles at 5875, 4471, 4713, 4922, and $5015 \AA$ were obtained for an $\mathrm{He} / \mathrm{H}$ ratio of $1.0(50 / 50)$ with an uncertainty of approximately \pm 0.2 .

To verify the model atmosphere parameters, we explore fits to the Paschen lines. The Balmer lines are affected by emission and blends with other lines for their line profiles to be useful as monitors of the atmospheric parameters, but the Paschen lines appear free of emission and blends. The Stark broadening tables for the Paschen lines of hydrogen are taken from Lemke (1997). In the lower panel of the Figure 1, we show observed and synthesized Paschen profiles near $8600 \AA$ for the model with He/ $\mathrm{H}=1.0$ and $T_{\text {eff }}=8000 \mathrm{~K}$ and for surface gravities $\log g=0.5$, 0.75 , and 1.0. The best-fitting profile is for $\log g$ of 0.75 . We also synthesized the Paschen lines in the $8400 \AA$ A region for the above mentioned temperature and gravities and we get consistent results. These profiles are also sensitive to the adopted effective temperature with a $1000 \mathrm{~K}$ increase approximately mimicking a 0.5 dex increase in surface gravity. The Paschen lines give the locus shown in the top panel of Figure 1. As the Paschen limit is approached, the Paschen lines overlap and depress the local continuum. In addition, the location of the continuum is rendered more challenging because of the echelle spectrometer's blaze function. Yet, the synthesized profiles closer to the limit are able to reproduce the observed profiles quite well—see bottom panel of Figure 1.

Our demonstration that SAO 40039 is H-deficient and $\mathrm{He}$-rich rests on the assumption that the $\mathrm{He} \mathrm{I}$ absorption lines originate in the stellar photosphere and are not a product of a stellar chromosphere. (A second assumption is that the real stellar photosphere approximates the model atmospheres computed by classical procedures.) A chromospheric origin is not trivially dismissed. One recalls that the D3 line appears in absorption in spectra of F dwarfs with active chromospheres (Wolff \& Heasley 1984; Danks \& Lambert 1985); the correlation between D3 equivalent width and X-ray luminosity points strongly to a chromospheric origin. A chromospheric origin for He absorption is required to account for the presence of He I $10830 \AA$ in spectra of warm-cool giants and supergiants where it may appear in absorption and/or emission (O'Brien \& Lambert 1986) and cannot be of photospheric origin.

The possibility of the photospheric origin of the He I lines in our spectrum is supported by several facts. First, the lines have the photospheric velocity. Second and more importantly, the equivalent widths of all $\mathrm{He}$ I lines - singlets and triplets - are reproduced by a photospheric model. If the lines originated in the chromosphere, one would expect a difference between singlets 


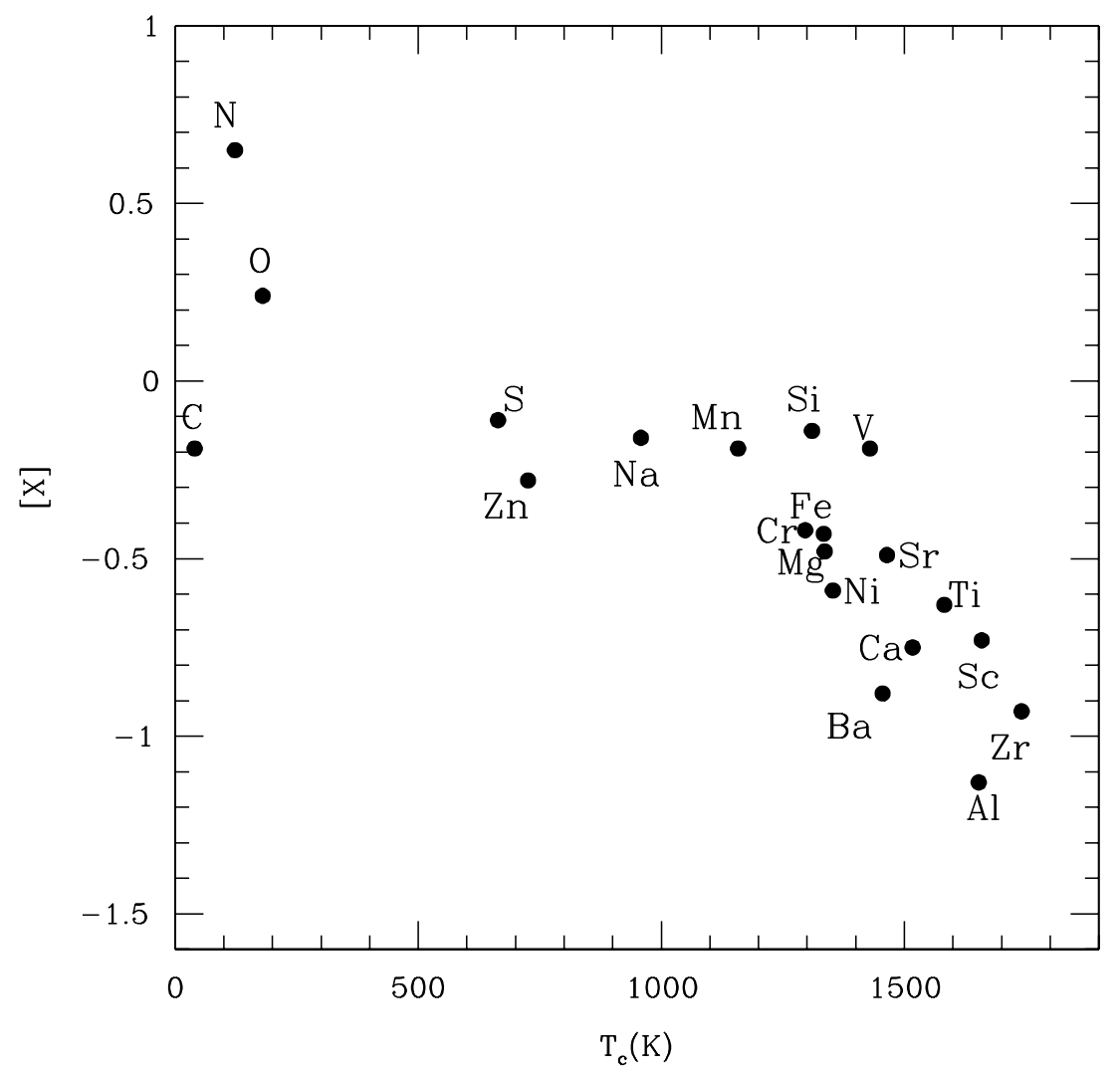

Figure 3. $[\mathrm{X}]$ vs. $T_{c}$ plot for SAO 40039.

and triplets, as previously noted by Klochkova et al. (2002) in their discussion of the He I absorption lines found in HD 331319 , a star similar to SAO 40039. While our analysis does not preclude a chromospheric origin, we agree with Klochkova et al. who remark that "the hypothesis of a photospheric origin for the He I lines in HD 331319 is not rejected." Certainly, there must be a suspicion that SAO 40039's atmosphere is mildly H-deficient such that now $\mathrm{He} / \mathrm{H}=1$, a value indicating appreciable loss of $\mathrm{H}$ and enrichment of $\mathrm{He}$ since the star was a main-sequence star with $\mathrm{He} / \mathrm{H} \simeq 0.1$.

\section{DISCUSSION AND RESULTS}

Using the adopted model parameters $\left(T_{\text {eff }}, \log g, \xi_{t}\right)=(8000$, $0.75,4.8$ ) with $\mathrm{He} / \mathrm{H}$ of 1.0 , the measured equivalent widths of different species were used to derive the abundances. In Table 1 we present the abundances derived with the SPECTRUM code using the $\mathrm{He} / \mathrm{H}=1.0$ model and the abundances generated with the code MOOG using the $\mathrm{He} / \mathrm{H}=0.1$ model.

A detailed line list (see Table 2, which lists some lines of SAO 40039) used in our analysis has been presented electronically. The line list provides the LEP $(\chi)$ for each line, the $\log g f$ value, sources of $\log g f$, the measured equivalent widths $\left(W_{\lambda}\right)$ in $\mathrm{m} \AA$, and the abundance $(\log \epsilon)$ derived from each line for the adopted model atmosphere.

The solar abundances for all the elements have been taken from Asplund et al. (2009).

SAO 40039 seems to be mildly iron-poor. Klochkova et al.'s analysis assuming $\mathrm{He} / \mathrm{H}=0.1$ gave a solar $\mathrm{Fe}$ abundance, a value essentially confirmed by our analysis for the model atmosphere also with $\mathrm{He} / \mathrm{H}=0.1$. A principal effect of the higher He abundance is to reduce the continuous opacity per
Table 2

Detailed Line List for SAO 40039

\begin{tabular}{|c|c|c|c|c|}
\hline Line & $\begin{array}{c}\chi \\
(\mathrm{eV})\end{array}$ & $\log g f$ & $\begin{array}{c}W_{\lambda} \\
(\mathrm{m} \AA)\end{array}$ & $\log \epsilon$ \\
\hline $\mathrm{He}_{\mathrm{I}} \lambda 5875.61$ & 20.96 & $+0.739^{a}$ & Synth & 11.45 \\
\hline He I $\lambda 4471.47$ & 20.96 & $+0.053^{\mathrm{a}}$ & Synth & 11.40 \\
\hline He I $\lambda 4921.93$ & 21.21 & $-0.435^{\mathrm{a}}$ & Synth & 11.45 \\
\hline Не г $\lambda 5015.67$ & 20.61 & $-0.819^{\mathrm{a}}$ & Synth & 11.50 \\
\hline $\mathrm{He}_{\mathrm{I}} \lambda 4713.13$ & 20.96 & $-0.975^{\mathrm{a}}$ & Synth & 11.40 \\
\hline C I $\lambda 4775.87$ & 7.49 & $-2.304^{\mathrm{a}}$ & 35 & 8.41 \\
\hline $\mathrm{C}_{\mathrm{I}} \lambda 5039.05$ & 7.48 & $-2.100^{\mathrm{a}}$ & 33 & 8.15 \\
\hline $\mathrm{C}_{\mathrm{I}} \lambda 4770.02$ & 7.48 & $-2.437^{\mathrm{a}}$ & 30 & 8.45 \\
\hline C I $\lambda 4766.67$ & 7.48 & $-2.400^{\mathrm{a}}$ & 26 & 8.34 \\
\hline С г $\lambda 4826.80$ & 7.49 & $-2.140^{\mathrm{a}}$ & 24 & 8.04 \\
\hline
\end{tabular}

Notes.

a NIST database (http://physics.nist.gov/PhysRefData/ASD/lines_form.html).

(This table is available in its entirety in a machine-readable form in the online journal. A portion is shown here for guidance regarding its form and content.)

gram and so demand a lower iron abundance to match the same observed line strength. Inspection of the $[\mathrm{X} / \mathrm{Fe}]$ entries in Table 1 points to several anomalies when the composition of an unevolved disk star is taken as the reference. The two most extreme anomalies are nitrogen which is very overabundant $([\mathrm{N} / \mathrm{Fe}]=+1.1)$, and aluminum which is markedly underabundant $([\mathrm{Al} / \mathrm{Fe}]=-0.7)$. Closer study shows that sodium may be overabundant $([\mathrm{Na} / \mathrm{Fe}]=+0.3)$ and a suite of elements are mildly underabundant: $\mathrm{Ca}, \mathrm{Sc}, \mathrm{Ti}, \mathrm{Zr}$, and $\mathrm{Ba}$ with $[\mathrm{El} / \mathrm{Fe}] \simeq-0.35$.

This abundance pattern is largely reminiscent of the pattern exhibited by stars affected by dust-gas separation, i.e., the 
photosphere is dominated by accretion of gas but not dust from a cool envelope, possibly a circumbinary dusty disk. Dust-gas separation is most remarkably exhibited by postAGB binaries such as HR 4049 (Van Winckel 2003). In such cases, the abundance anomalies are correlated with the condensation temperature $\left(T_{c}\right)$ : elements with high $T_{c}$ are most underabundant. In Figure 3 , we plot $[\mathrm{X}]$ (where $[\mathrm{X}]=$ $\left.\log \epsilon(\mathrm{X})_{*}-\log \epsilon(\mathrm{X})_{\odot}\right)$ versus the $T_{c}$ computed by Lodders (2003) for a solar composition mixture. Elements $\mathrm{N}$ and $\mathrm{O}$ depart from a general tendency for $[\mathrm{X}]$ to decrease with increasing $T_{c}$. In presenting Figure 3 , the adopted reference has been that $[\mathrm{X} / \mathrm{Fe}] \simeq 0.0$ for all elements in an unevolved star with $[\mathrm{Fe} / \mathrm{H}] \simeq-0.4$. This is essentially true for a thin disk star, although a dispersion in heavy elements at a given $[\mathrm{Fe} / \mathrm{H}]$ was noted by Edvardsson et al. (1993). If SAO 40039 is a thick disk star, the $\alpha$-elements in Figure 3 should be plotted with [X] about -0.2 dex less than shown in the figure but the overall trend and the scatter about that trend will be unaffected. The $\mathrm{Na}, \mathrm{S}$, and $\mathrm{Zn}$ abundances suggest that the initial metallicity of the star was $[\mathrm{Fe} / \mathrm{H}]_{\mathrm{o}} \simeq-0.2$.

The high $\mathrm{N}$ abundance is, probably, a consequence of extensive burning of $\mathrm{H}$ to $\mathrm{He}$ by the $\mathrm{CN}$ cycle with concomitant conversion of $\mathrm{C}$ to $\mathrm{N}$. Survival of $\mathrm{C}$ at about its initial abundance suggests that $\mathrm{N}$ synthesis occurred primarily after the atmosphere was enriched with $\mathrm{C}$ at the third dredge-up. One possibility is $\mathrm{H}$-consumption during a final $\mathrm{He}$-shell flash (Herwig 2001; Blöcker 2001). The O is overabundant with respect to the initial abundance, possibly it was enriched by the third dredge-up and temperatures during the late episode of $\mathrm{H}$-burning were too low to destroy $\mathrm{O}$, or non-LTE line formation can reduce the $\mathrm{C}, \mathrm{N}$, and $\mathrm{O}$ abundances. For an effective temperature of about $8000 \mathrm{~K}$, Venn (1995) gives non-LTE correction to $\mathrm{C}$ and $\mathrm{N}$ abundances from $\mathrm{C}$ I and $\mathrm{N}$ I lines of about -0.4 dex for normal supergiants. Takeda \& Takada-Hidai (1998) have estimated NLTE corrections for O I to be -0.1 dex at this effective temperature for supergiants. After applying these non-LTE corrections, the above sketch of a possible origin for SAO 40039 is little affected.

\section{CONCLUDING REMARKS}

Though the existence of extremely H-deficient stars with $\mathrm{He} / \mathrm{H}$ ratio of about 10,000 , showing He I lines in their absorption spectra, is known (Pandey et al. 2001; Pandey \& Reddy 2006), SAO 40039, from our estimated $\mathrm{He} / \mathrm{H}$ ratio of about unity, appears to be the first detection of a mildly H-deficient star. Lambert (1996) had commented on the possibilities of such stars and on the difficulty of detecting stars too cool to show He I lines. With an effective temperature of about $8000 \mathrm{~K}$, SAO 40039 seems to be responding to this call.

It is very likely that other post-AGB stars may belong to the mildly H-deficient class. Several examples like SAO 40039 have detectable He I lines. The question then arises; are post-AGB stars of a temperature too low to provide $\mathrm{He}$ I lines also He-rich and how can their H-deficiency be unmasked? Exploration of post-AGB stars showing He I lines needs to be continued with models of appropriate $\mathrm{H} / \mathrm{He}$ ratios. Additionally, non-LTE abundance analyses as described by Pandey \& Lambert (2011) should be performed to further refine knowledge of the chemical compositions.

We are thankful to the VBO observing support team, in particular to G. Selvakumar, for obtaining a spectrum of SAO 40039 using the VBT echelle spectrometer. D.L.L. thanks the Robert A. Welch Foundation of Houston, Texas for support through grant F-634.

\section{REFERENCES}

Asplund, M., Grevesse, N., Sauval, A. J., \& Scott, P. 2009, ARA\&A, 47, 481 Blöcker, T. 2001, Ap\&SS, 275, 1

Danks, A. C., \& Lambert, D. L. 1985, A\&A, 148, 293

Edvardsson, B., Andersen, J., Gustafsson, B., et al. 1993, A\&A, 275, 101

Fujii, T., Nakada, Y., \& Parthasarathy, M. 2002, A\&A, 385, 884

Herwig, F. 2001, Ap\&SS, 275, 15

Jeffery, C. S., Woolf, V. M., \& Pollacco, D. L. 2001, A\&A, 376, 497

Klochkova, V. G., Chentsov, E. L., Tavolzhanskaya, N. S., \& Panchuk, V. E. 2007, Astron Rep., 51, 642

Klochkova, V. G., Panchuk, V. E., \& Tavolzhanskaya, N. S. 2002, Astron. Lett., 28, 49

Lambert, D. L. 1996, in ASP Conf. Ser. 96, Hydrogen Deficient Stars, ed. C. S. Jeffery \& U. Heber (San Francisco, CA: ASP), 443

Lemke, M. 1997, A\&AS, 122, 285

Lodders, K. 2003, ApJ, 591, 1220

O’Brien, G. T., Jr., \& Lambert, D. L. 1986, ApJS, 62, 899

Pandey, G. 2006, ApJ, 648, L143

Pandey, G., \& Lambert, D. L. 2011, ApJ, 727, 122

Pandey, G., Rao, N. K., Lambert, D. L., Jeffery, C. S., \& Asplund, M. 2001, MNRAS, 324, 937

Pandey, G., \& Reddy, B. E. 2006, MNRAS, 369, 1677

Rao, N. K., Sriram, S., Jayakumar, K., \& Gabriel, F. 2005, JA\&A, 26, 331

Sneden, C. 1973, PhD thesis, Univ. of Texas at Austin, USA

Takeda, Y., \& Takada-Hidai, M. 1998, PASJ, 50, 629

Tull, R. G., MacQueen, P. J., Sneden, C., \& Lambert, D. L. 1995, PASP, 107, 251

Van Winckel, H. 2003, ARA\&A, 41, 391

Van Winckel, H., Waelkens, C., \& Waters, L. B. F. M. 1996, A\&A, 306, L37

Venn, K. A. 1995, ApJ, 449, 839

Waelkens, C., Van Winckel, H., Trams, N. R., \& Waters, L. B. F. M. 1992, A\&A, 256, L15

Wolff, S. C., \& Heasley, J. N. 1984, PASP, 96, 231 